\title{
Modified-AND Subtraction Detection Technique Based on Weight-Division for SAC-OCDMA Systems
}

\author{
Hamza M. R. Al-Khafaji, S. A. Aljunid, and Hilal A. Fadhil
}

\begin{abstract}
In this paper, we investigate the use of the modified-AND subtraction detection to assuage the intensity noise in spectral-amplitude coding optical code-division multiple-access (SAC-OCDMA) systems. This detection approach is based upon reducing the received power to the decoders by dividing the weight of the utilised code sequence. The proposed technique has the ability to suppress the influence of multiple-access interference (MAI), in addition to alleviate the intensity noise effect. We simulate the system performance of the proposed detection technique using OptiSystem software Version 9.0. The software considered the entire practical impacts in the system, such as nonlinear effect, dispersion, and attenuation in the fiber. The simulation results based on the modified quadratic congruence (MQC) code show that the modified-AND subtraction detection offer best bit-error rate (BER) performance compared to traditional AND subtraction detection.
\end{abstract}

Index Terms-Optical code-division multiple-access, spectral-amplitude coding (SAC), Modified-AND subtraction detection, multiple-access interference, intensity noise.

\section{INTRODUCTION}

Optical code-division multiple-access (OCDMA) has attracted great attention, due to its desirable features, such as asynchronous access, soft-capacity, good security and so on [1], [2]. It takes advantage of sporadic use of channels by allowing simultaneous connection of multiple users to the network [3]. Therefore, it has been proposed for flexibly implementing the optical access networks, such as passive optical networks (PONs), in current and future communication and computer networks [4]. On the contrary, the influence of MAI is considered as the main reason of performance degradation in conventional OCDMA systems [5]. In MAI environments, each receiver receives all the signals from all active encoders in OCDMA system. While the matching channel signal is recovered by a decoder, all other unmatched channel signals play the role of noise, which can produce MAI noise [6]. Among all OCDMA techniques, SAC has the advantage of eliminating the effect of MAI by using subtraction detection techniques at the receiver side with fixed in-phase cross-correlation codes [7].

Incoherent broadband sources such as light emitting diodes (LEDs) or an erbium doped fiber source are the most obvious choice in SAC-OCDMA system because they are low-priced as compared to coherent sources [8], [9].

Manuscript received October 19, 2012; revised November 29, 2012

The authors are with the School of Computer and Communication Engineering, University Malaysia Perlis (UniMAP), Perlis, Malaysia; Corresponding author: Hamza M. R. Al-Khafaji (e-mail: eng.hamza.alkhafaji@gmail.com).
However, the intensity noise considered as the main limitation of these sources. This noise is power dependent so that increasing the signal strength at the detection side does not improve the signal-to-noise ratio (SNR) [10]. As such, the need of an appropriate detection technique compatible with fixed in-phase cross-correlation codes to lessen the influences of intensity noise is justified.

Among SAC codes, MQC $\left(L=p^{2}+p, K=p^{2}, w=p+1, \lambda\right.$ $=1$ ) is parameterized with a prime number $p$ and is characterized by unity cross-correlation value yields an ideal cross-correlation [11], [12], where $L$ is the code length, $K$ is the number of users, $w$ is the code weight, and $\lambda$ is the cross-correlation value between any two codes of the family. The residue of this paper is organised as follows. Section II presents the design of modified-AND subtraction detection technique. Next, in Section III we simulate the SAC-OCDMA system using and compare the resultant eye diagrams. And finally, the paper is concluded in Section IV.

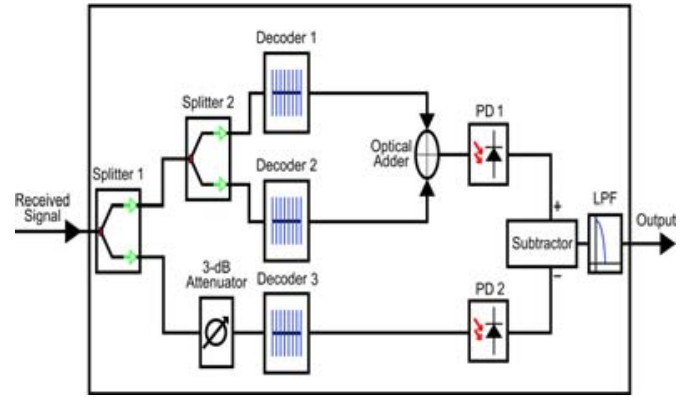

Fig. 1. SAC-OCDMA receiver based on modified-AND subtraction detection technique.

\section{MODIFIED-AND SUBTRACTION DETECTION}

The SAC-OCDMA receiver diagram of this technique is depicted in Fig. 1 [13], [14]. The received optical power is split by splitter 1 into two parts; one to the upper decoding branches (decoder 1 and decoder 2) and the other to the decoder 3 through an attenuator. The 3 -dB attenuator ensures that the interference signal has an equal power incident on each photo-detector in the case of inactive user. The upper decoders (decoder 1 and decoder 2) are placed in a parallel configuration to divide the weight of the used code and reduce the optical power level during the decoding process. The upper decoders have a spectral response match with the active user, while decoder 3 has the overlapped bins from different interferers. These overlapped bins can be represented mathematically by AND operation between the active user and interferers [15]-[17]. This technique is implemented by using the fiber Bragg-gratings (FBGs) to decode the received signal due to its low insertion losses, good spectral resolution and low material costs [18]. The 
photo-detector is composed of two photodiodes (PD 1 and PD 2), which are connected electrically in opposition to provide an electrical signal that is proportional to the power difference of the two optical inputs. The equivalent passband resulting from the filtering is chosen to equal $0.75 \times R_{B}$, where $R_{B}$ is the bit rate.

\section{Simulation SETUP AND RESUltS}
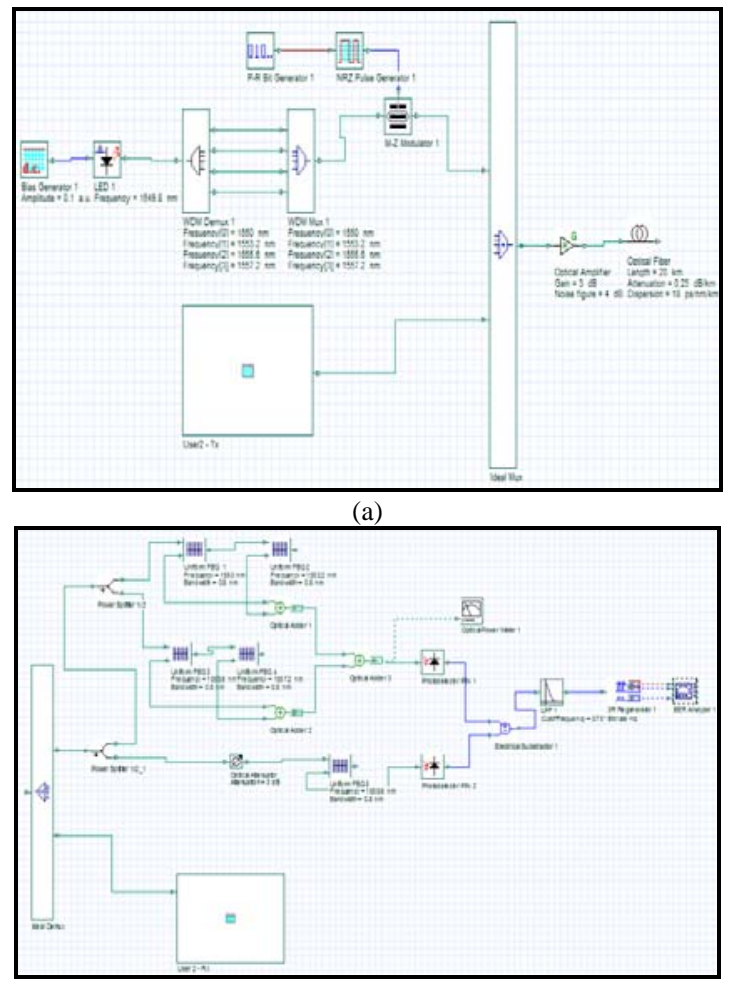

(b)

Fig. 2. Simulation setup of SAC-OCDMA using modified-AND subtraction detection technique; (a) Transmitter; (b) Receiver.

The system was designed and simulated using OptiSystem software Version 9.0, which is widely used for optical fiber simulations. A simple schematic diagram for two users is illustrated in Fig. 2. The simulation was carried out using MQC code (Weight, $w=4$ ) for up to five active users at the bit rate of each user $R_{B}=622 \mathrm{Mb} / \mathrm{s}$ (STM-4). The ITU-T G.652 standard single-mode optical fiber is employed for a $20 \mathrm{Km}$ optical transmission. Each chip has a spectral width of $0.8 \mathrm{~nm}$. The attenuation and dispersion were set at $0.25 \mathrm{~dB} / \mathrm{km}$ and 18 $\mathrm{ps} / \mathrm{nm} \cdot \mathrm{km}$, respectively. The nonlinear effects were activated and specified according to the typical industrial values to simulate the real environment as close as possible. The noise generated at the receivers was set to be random and totally uncorrelated. The dark current value was set at $5 \mathrm{nA}$, and the thermal noise coefficient was $18 \times 10^{-24} \mathrm{~W} / \mathrm{Hz}$ for each of the photo-diodes at the detection part. The performance of the system was characterized by referring to the BER and eye pattern. Further, it will be assumed that the transmitted power of the light source is limited. However, to overcome this limitation an erbium doped fiber amplifier (EDFA) is used with optimum EDFA gain of $3 \mathrm{~dB}$. The eye pattern of three users for the modified-AND subtraction detection is shown in Fig. 3. It can be seen from Fig. 3 that the average BER value of the three users is $1.63 \times 10^{-18}$, while it is $1.15 \times 10^{-16}$ for the previous AND subtraction detection. Fig. 4 shows the system performance in terms of BER for MQC code using both detection techniques. It is clearly seen from Fig. 4 that the performance of modified-AND subtraction detection is much better compared to AND subtraction detection.

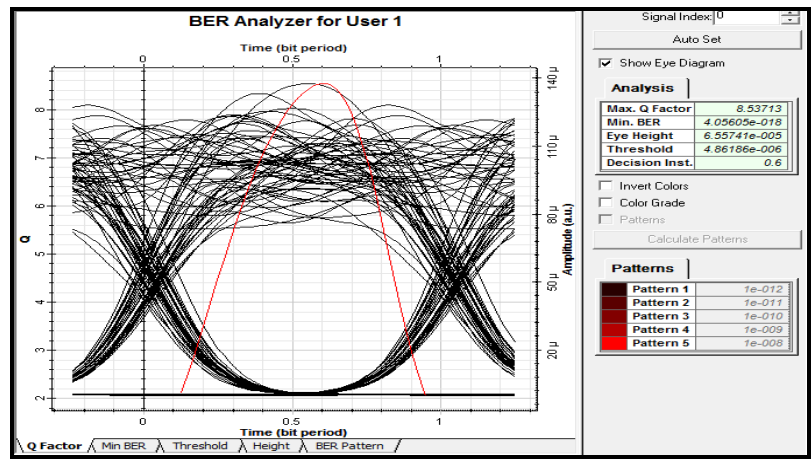

(a)

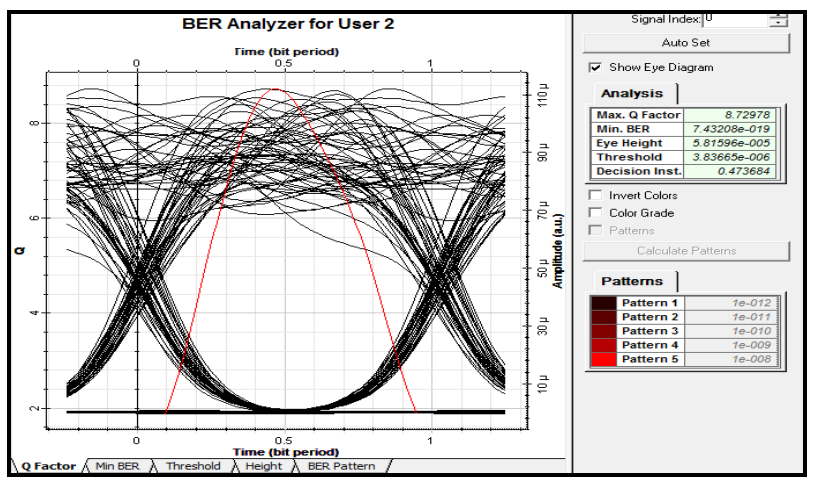

(b)

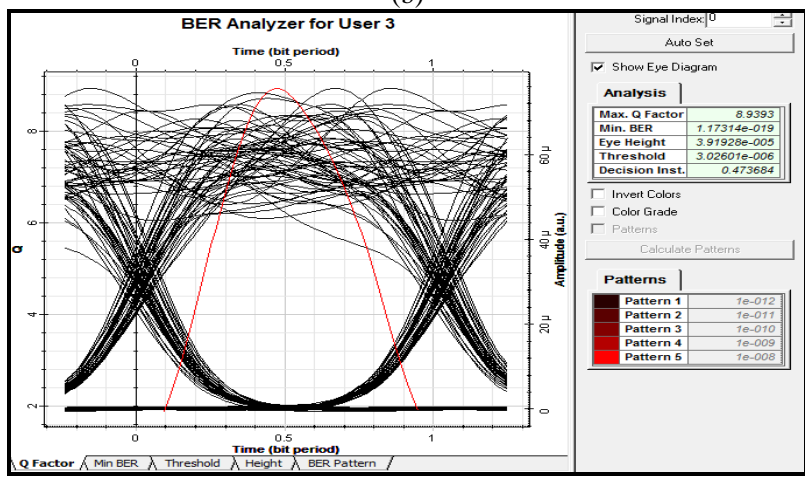

(c)

Fig. 3. Eye diagram results for three active users of MQC code using modified-AND subtraction detection.

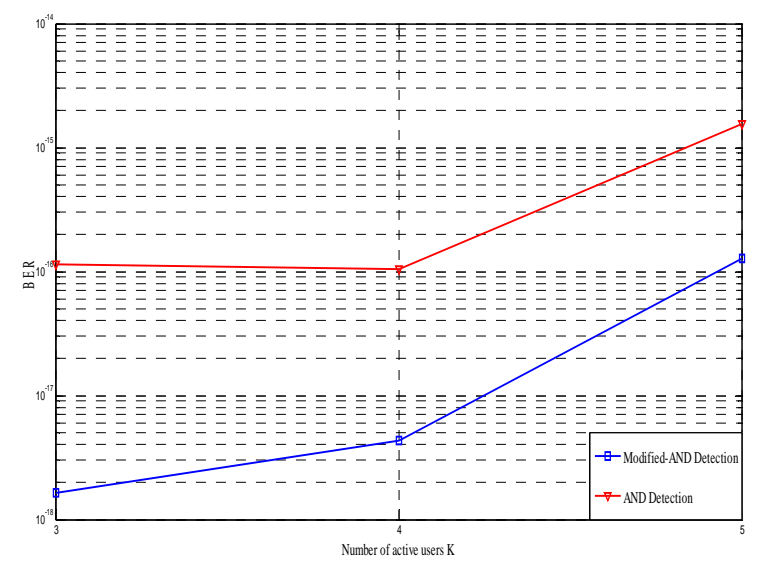

Fig. 4. BER variation against the number of active users for both detection techniques using MQC code. 


\section{CONCLUSIONS}

We have presented a modified-AND subtraction detection for SAC-OCDMA systems to eliminate the MAI effect and mitigate the intensity and shot noise impacts. This technique is based on decreasing the received signal strength to the decoding branches by dividing the spectrum of the utilised code sequence. In order to simulate the real environment as close as possible, the influences of fiber nonlinearities, dispersion and attenuation were considered in the simulation according to the typical industrial values. We ascertained that the modified-AND subtraction detection improves SAC-OCDMA system performance significantly as compared with the conventional AND subtraction detection. An interesting direction for future research lies in analysing the spectral efficiency and BER performance related to the new proposed technique using different SAC codes.

\section{REFERENCES}

[1] F. A. Aziz and S. S. A. Obayya, "Manchester-coded modified-legendre codes for spectral-amplitude coding-based optical code-division multiplexing system," IET Optoelectron., vol. 5, pp. 93-98, 2011.

[2] S. Sun and M. S. Leeson, "Higher-order dispersion mitigation for spectrum-sliced FFH-OCDMA using adaptive prime-hop codes," Photon. Netw. Commun, vol. 21, pp. 107-116, 2011.

[3] M. Rochette, S. Ayotte, and L. A. Rusch, "Spectral efficiency of frequency-encoded OCDMA systems with incoherent sources," in Proc. of $30^{\text {th }}$ Eur. Conf. Opt. Commun. (ECOC 2004), Sep. 2004.

[4] S. Khaleghi, M. R. Pakravan, "Quality of service provisioning in optical CDMA packet networks,” J. Opt. Commun. Netw, vol. 2, pp. 283-292, May 2010.

[5] W. J. Huang, C. T. Niu, C. H. Lin, and J. Wu, "Spatial/spectral OCDMA system using partial modified prime codes and error-correction codes," J. Lightwave Technol, vol. 26, pp. 3030-3040, September 2008.

[6] S. H. Chang, B. K. Kim, H. Park, W. K. Lee, and K. Kim, "Performance of bipolar optical spectral encoding CDMA with modified PN codes," ETRI J., vol. 28, pp. 513-516, 2006.

[7] M. Noshad, and K. Jamshidi, "Code family for modified spectral-amplitude-coding OCDMA systems and performance analysis," J. Opt. Commun. Netw, vol. 2, pp. 344-354, June 2010.

[8] E. D. J. Smith, R. J. Blaikie, and D. P. Taylor, "Performance enhancement of spectral-amplitude-coding optical CDMA using pulse-position modulation," IEEE Trans. Commun., vol. 46, pp. 1176-1185, September 1998.

[9] M. Rochette, S. Ayotte, and L. A. Rusch, "Analysis of the spectral efficiency of frequency-encoded OCDMA systems with incoherent sources,” J. Lightwave Technol., vol. 23, pp. 1610-1619, April 2005.

[10] S. Ayotte, M. Rochette, J. Magne, L. A. Rusch, and S. LaRochelle, "Experimental verification and capacity prediction of FE-OCDMA using superimposed FBG,” J. Lightwave Technol., vol. 23, pp. 724-731, February 2005.

[11] Z. Wei, and H. G. Shiraz, "Codes for spectral-amplitude-coding optical CDMA systems,” J. Lightwave Technol., vol. 20, pp. 1284-1291, August 2002.

[12] Z. Wei, H. M. H. Shalaby, and H. G. Shiraz, "Modified quadratic congruence codes for fiber bragg-grating-based

spectral-amplitude-coding optical CDMA systems,” J. Lightwave Technol., vol. 19, pp. 1274-1281, September 2001.

[13] H. M. R. A. Khafaji, S. A. Aljunid, and H. A. Fadhil, "Improved BER based on intensity noise alleviation using developed detection technique for incoherent SAC-OCDMA systems," J. of Modern Optics, 2012.

[14] H. M. R. A. Khafaji, S. A. Aljunid, and H. A. Fadhil, "Performance enhancement of SAC-OCDMA system using modified-AND subtraction detection," in Proc. of 2011 IEEE Conference on Computer Applications and Industrial Electronics (ICCAIE 2011), no. 6162170, pp. 412-415, 2011.

[15] S. A. Aljunid, M. Ismail, A. R. Ramli, B. M. Ali, and M. K. Abdullah, "A new family of optical code sequences for spectral-amplitude-coding optical CDMA systems," IEEE Photon. Technol. Lett., vol. 16, pp. 2383-2385, October 2004.

[16] F. N. Hasoon, S. A. Aljunid, M. D. A. Samad, M. K. Abdullah, and S. Shaari "Spectral amplitude coding OCDMA using AND subtraction technique," Applied Optics, vol. 47, pp. 1263-1268, 2008.

[17] I. S. Hmud, F. N. Hasoon, and S. Shaari, "Optical CDMA system parameters limitations for AND subtraction detection scheme under enhanced double weight (EDW) code based on simulation experiment," Opt. Appl., vol. 40, pp. 669-676, 2010.

[18] L. A. Rusch, "Theoretical and practical capacity of OCDMA using spectral amplitude coding," in Proc. of SPIE - The International Society for Optical Engineering 5970 II , art. no. 59702B, 2005.

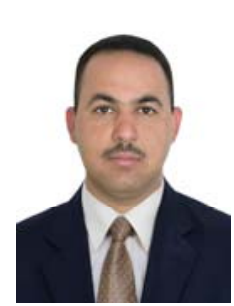

Hamza Mohammed Ridha Al-Khafaji received the B.Sc. degree in electronic and communications engineering and the M.Sc. degree in communications engineering from Al-Nahrain University, Baghdad, Iraq, in 2004 and 2007 respectively. He is currently a Ph.D. communication engineering student at University Malaysia Perlis (UniMAP), school of computer and communication engineering. He has working experience as a senior BSS engineer in Huawei Technologies and Omnnea Wireless Telecom, Iraq from 2006 until 2010. His research interest's are in OCDMA, wireless networks and mobile communications.

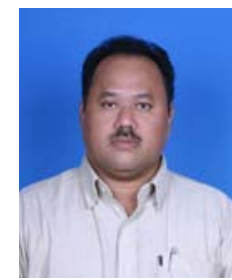

multiplexing.

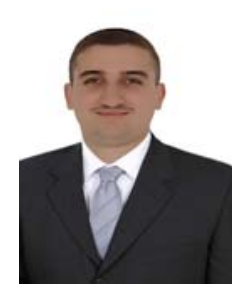
interests include OCDMA technologies and wavelength division multiplexing
Syed Alwee Aljunid received the BEng in computer and communication systems (first-class honors) and the Ph.D. degree in communication and network and 2005, respectively. He is currently a professor in the School of Computer and Communication Engineering, University Malaysia Perlis (UniMAP), Perlis, Malaysia. His research interests include OCDMA technologies and wavelength division

Hilal Adnan Fadhil received the B.Sc. in electronic and communications engineering from Al-Nahrain University in 2002, M.Sc. degree in communications engineering from Al-Nahrain University in 2004, and Ph.D. degree in optical communications from University Malaysia Perlis in 2010. He is currently a senior lecturer in the School of Computer and Communication Engineering, University Malaysia Perlis (UniMAP), Perlis, Malaysia. His research engineering from University Putra Malaysia in 2001 